\title{
Motion Correction in Respiratory Gated Cardiac PET/CT Using Multi-scale Optical Flow
}

\author{
Mohammad Dawood ${ }^{1,3}$, Thomas Kösters ${ }^{2}$, Michael Fieseler ${ }^{1}$, \\ Florian Büther ${ }^{3}$, Xiaoyi Jiang ${ }^{1}$, Frank Wübbeling ${ }^{2}$, and Klaus P. Schäfers ${ }^{3}$ \\ ${ }^{1}$ Department of Computer Science, University of Münster, Germany \\ dawood@uni-muenster. de \\ ${ }^{2}$ Department of Nuclear Medicine, University Hospital Münster, Germany \\ ${ }^{3}$ Department of Mathematics, University of Münster, Germany
}

\begin{abstract}
Respiratory motion is a source of degradation in positron emission tomography. As the patients cannot hold breath during the PET acquisition, spatial blurring and motion artifacts are unavoidable which may lead to wrong quantification of the data. A solution based on respiratory-gating and optical flow based correction of the PET data is proposed. This includes deformation of the CT data for accurate attenuation and listmode based reconstruction. All methods are applied to real patient data and are evaluated with respect to three criteria.
\end{abstract}

\section{Introduction}

Respiratory motion is a well known source of degradation in positron emission tomography (PET). Since cardiac PET studies typically require several minutes of acquisition time, the patients cannot hold their breath during the acquisition and respiratory motion is the result. Motion in PET studies leads to two problems: wrong attenuation correction and image blurring. Attenuation correction is required to account for the effects of photon absorption in the body. This problem is enhanced if the computed tomography (CT) data is also used for attenuation correction as in PET/CT. The CT represents a snapshot in comparison to the PET images thus the PET data is not always in spatial correspondence with the CT data and will be consequently wrongly corrected for attenuation. Motion also leads to image blur and may cause wrong staging [1, inaccurate localization [2] and wrong quantification [3] of the lesions. Most recent studies related to this problem estimate the respiratory motion on the high resolution and less noisy gated CT images [7, 8], 9]. This goes hand in hand with an increase in radiation dose for the patient, which is not justifiable for most cardiac patients. Using the PET data is difficult as it has low resolution and high noise.

A solution to this problem is proposed along the following lines: 1) The patient data is acquired on the PET/CT scanner using respiratory gating. 2) The respiratory motion is estimated with the help of a discontinuity-preserving optical flow algorithm. This algorithm is robust in presence of noise and usage of a multi-scale approach allows it to also accurately estimate large displacements.

D. Metaxas et al. (Eds.): MICCAI 2008, Part II, LNCS 5242, pp. 155 162, 2008.

(C) Springer-Verlag Berlin Heidelberg 2008 
3) The CT data is deformed to correspond to the different respiratory phases for accurate attenuation correction. 4) The PET data is reconstructed using the motion estimate and the deformed CT data in a single step 9].

\section{Respiratory Gating}

Respiratory gating is the method of dividing the PET data so that each part represents only a fraction of the total respiratory motion. The respiratory signal was acquired by laying a belt around the patient's waist with a marker attached to it. The marker was tracked with the help of a video camera as described in [5. The respiratory signal is than divided into eight parts with respect to the amplitude and used for reconstruction. Each image volume was reconstructed without attenuation correction, since that leads to artifacts on the images [10]. The images were reconstructed using a 3D OSEM algorithm [11. The reconstructed volumes had the dimensions $175 \times 175 \times 47$ and voxel size $3.375 \mathrm{~mm}$ in each direction.

\section{Discontinuity Preserving Optical Flow}

Optical flow methods calculate the motion between two image frames. As a voxel with intensity $I(x, y, z, t)$ moves a small distance between the two frames, the intensity of the pixel remains unchanged thus:

$$
I_{x} u+I_{y} v+I_{z} w=-I_{t}
$$

with $u, v, w$ for the $x, y$ and $z$ components of the velocity or optical flow and $I_{x}, I_{y}, I_{z}, I_{t}$ for the derivatives of the intensity image in corresponding directions respectively time. This basic equation contains three unknowns. Thus it needs to be supplied with additional conditions for obtaining a solution. Recently, a locally-consistent and globally-smooth optical flow method was proposed [12. A discontinuity-preserving (DP) algorithm is proposed here, which is based upon this method and has been adapted for application to $3 \mathrm{D}$ medical imaging [4]. The flow is found by minimizing:

$$
f_{D P}=\int\left\{\psi_{1}(D)+\alpha \psi_{2}(S)\right\} \mathrm{d} x \mathrm{~d} y \mathrm{~d} z
$$

where $D=k * V^{T}\left(\nabla I \nabla I^{T}\right) V$ gives the locally consistent term, $k$ is a gaussian kernel, and $S=|\nabla V|^{2}$ is the global smoothing term. $V=\left(V_{x}, V_{y}, V_{z}, 1\right)^{T}$ is the motion vector and $\nabla I=\left(I_{x}, I_{y}, I_{z}, I_{t}\right)^{T}$ lastly, $\psi_{i}$ are given by the functions:

$$
\psi_{i}\left(s^{2}\right)=2 \beta_{i}^{2} \sqrt{1+\frac{s^{2}}{\beta_{i}^{2}}}, i \in 1,2
$$

with $\beta_{i}$ as scaling factors. Discontinuity preservation is achieved by weighting the $\psi_{2}$ function, i.e. the smoothness in flow, in accordance with the magnitude 


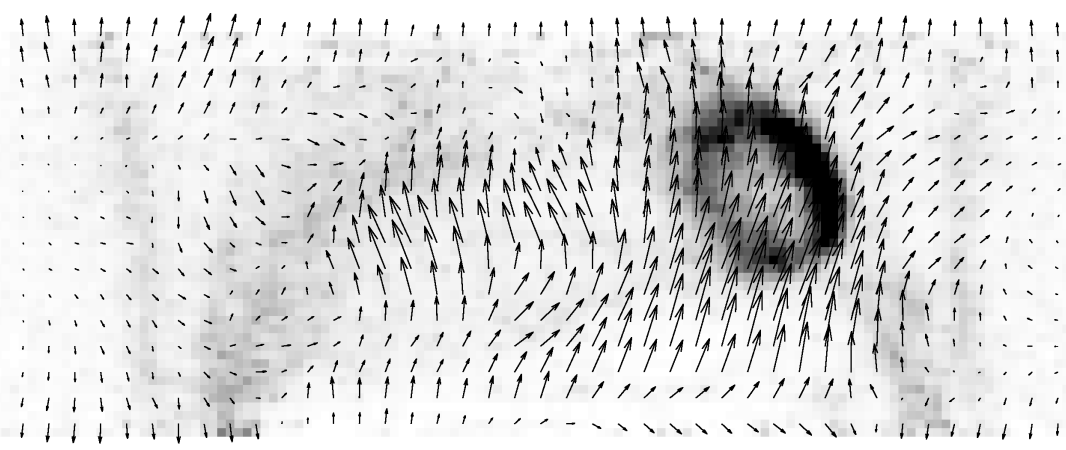

Fig. 1. Motion estimated on PET data. A coronal-slice from the 3D PET volume is shown overlaid with the estimated motion vectors.

of the edge at the voxel $i$. Thus areas with no or only weak edges are strongly smoothed as compared to areas with strong edges. Each component of the flow vector is separately weighted to make the algorithm anisotropic. An example of the estimated motion vectors is shown in Fig. 11 The values for the parameters were selected based on the optimization with the genetic algorithms toolbox by Matlab Inc. The typical values for these images were around 300 for $\alpha$, and 5.9 for $\beta_{1}$ and 5.2 for $\beta_{2}$. The number of inner iterations was around 200. The parameters are relatively stable with no apparent change in results for a range of values, e.g. $\alpha$ could be varied between 200 and 450 without any visible effect on the results.

The algorithm so far is robust enough to be used with noisy images. However, it becomes inaccurate with increasing motion. It is therefore proposed to calculate the flow in several small steps. The remaining motion at each step is corrected at the next higher level of resolution, thus:

$$
f\left(I_{o}\right)=f\left(I_{l}\right)+f\left(I_{h}\right)
$$

where $f\left(I_{o}\right)$ denotes the motion on original images, $f\left(I_{l}\right)$ denotes the motion estimated at a lower resolution, and $f\left(I_{h}\right)$ denotes the remaining motion present on the images in original resolution, already corrected for $f\left(I_{l}\right)$. Three levels of resolution were used, counting the original images as level one, in this study.

\section{Accurate Attenuation Correction}

Attenuation correction is needed for quantification of the PET data as it corrects the data for the effects of absorption of the gamma quants inside the body. In $\mathrm{PET} / \mathrm{CT}$ scanners, the CT scan is used for attenuation correction. The CT is acquired very fast and under breath-hold condition thus, there is a shift between $\mathrm{CT}$ and different PET respiratory phases. To solve this problem the motion field derived from the PET data is used to deform the CT data so that it corresponds to the PET data in all respiratory phases, see Fig. 2 Attenuation correction 


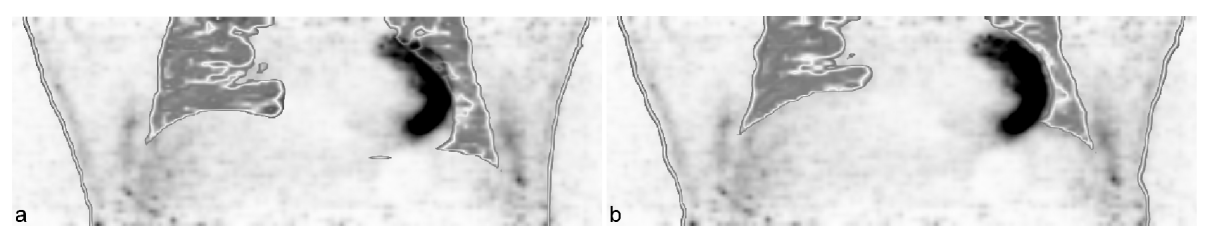

Fig. 2. CT data overlaid on the PET data. a) The CT does not corresponds to the respiratory phase of the $\mathrm{PET}$ data in the heart region. b) After motion correction, the CT data corresponds to the PET data.

performed with the deformed CT data is more accurate and avoids attenuation artifacts.

\section{Data Acquisition}

Seven patients with coronary artery disease were included in this study. A listmode dataset was acquired for 20 minutes, 1 hour post injection of ${ }^{18} \mathrm{FDG}$ (4 $\mathrm{MBq} / \mathrm{kg}$ ). The Siemens Biograph Sensation 16 PET/CT scanner (Siemens Medical Solution) with a dedicated listmode research package was used for data acquisition. The respiratory signal was acquired during the PET listmode acquisition. The PET scanner has an intrinsic spatial resolution of around $6 \mathrm{~mm}$ full-width-at-half-maximum (FWHM) 13 .

\section{Evaluation Criteria}

Three different method of evaluation were used to assess the performance of the motion correction scheme.

Motion of Heart. The heart moves due to the respiratory motion of the lungs and diaphragm. The reduction of heart motion, therefore, can be used as a measure for assessing the performance of the motion correction technique. A manually selected threshold was used to segment the heart on the PET images. The displacement was calculated by observing the motion of the center of mass of the segmented heart. These results take the 3D aspect of the PET data into account and are in absolute units (millimeters).

Correlation Coefficient. The second measure used is the correlation coefficient(CC). The correlation coefficient considered all voxels in the 3D volume. It is more prone to noise than the displacement of heart. The $\mathrm{CC}$ is a global indication of the performance of the motion correction method.

Myocardial Thickness. Myocardial activity is spread across a region due to the motion of the heart which leads to an apparent thickening of the myocardium. Thicker myocardium thus indicates motion of heart. Three consecutive transaxial slices from the center of the heart were manually selected on each patient data. 
The myocardial thickness was measured on the PET data by fitting a gaussian function to the anterior wall of the myocardium and calculating it's FWHM.

\section{Results}

Motion of Heart. The results of the motion of the heart criteria are summarized in Table 1. The range of motion on the uncorrected data was $21.6 \mathrm{~mm}$ to $6.6 \mathrm{~mm}$ for different patients, whereas the corrected data shows a range of only $0.5 \mathrm{~mm}$ to $0.2 \mathrm{~mm}$. Fig. 1 shows the estimated motion vectors on a single coronal-slice taken from the 3D PET volume. It demonstrates that the main motion is present in the heart region and adjacent lung base, this corresponds with the expectations. Also, that the motion discontinuities are preserved strongly along the organ boundaries. The final result of the motion correction scheme is shown in Fig. 3 .

Correlation Coefficient. The correlation coefficient analysis shows the same results as the motion of the heart criteria (Table 2). The values are shown only for the Gates 1, 4, and 8 to report condensed results. Gate 1 being the target gate, the correlation for Gate 1 is naturally overall $100 \%$.

Myocardial Thickness. The results of the myocardial thickness assessment are given in Table 3. The thickness of the anterior wall varies from patient to

Table 1. Motion of Heart on Patient Data [mm]

\begin{tabular}{l|rr} 
Patient & Original After correction \\
\hline P1 & 21.6 & 0.2 \\
P2 & 8.4 & 0.2 \\
P3 & 12.0 & 0.5 \\
P4 & 13.4 & 0.2 \\
P5 & 8.9 & 0.3 \\
P6 & 6.6 & 0.2 \\
P7 & 6.7 & 0.3
\end{tabular}

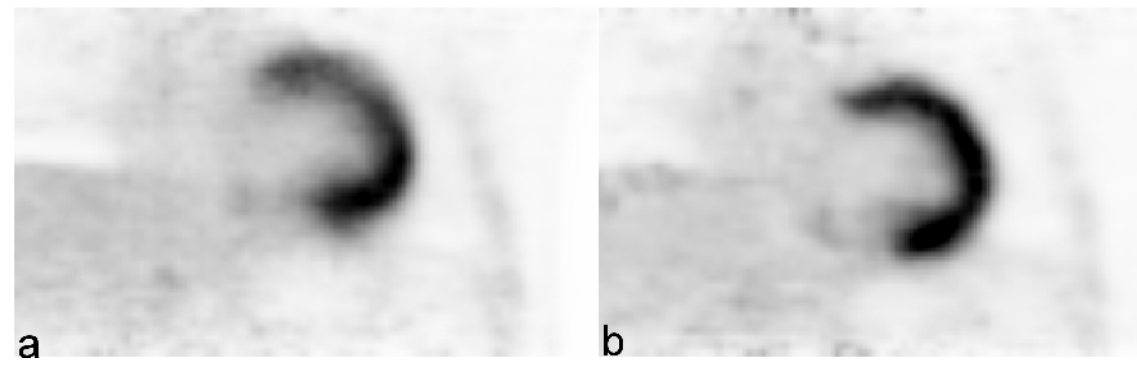

Fig. 3. a) Ungated PET/CT images after attenuation correction. b) The same data after attenuation and motion correction. The blur and the apparently decreased tracer uptake of the anterior wall on a) is corrected in b). 
Table 2. Correlation Coefficient Analysis on Patient Data, [\%]

\begin{tabular}{l|lll|l} 
Patient & Gate 1 Gate 4 Gate 8 & Average \\
\hline P1 Original & 100.00 & 73.09 & 61.30 & $78.13 \pm 20$ \\
P1 Corrected & 100.00 & 94.13 & 93.78 & $95.97 \pm 3$ \\
P2 Original & 100.00 & 88.65 & 80.00 & $89.55 \pm 10$ \\
P2 Corrected & 100.00 & 95.12 & 94.95 & $96.69 \pm 3$ \\
P3 Original & 100.00 & 84.51 & 80.34 & $88.28 \pm 10$ \\
P3 Corrected & 100.00 & 92.81 & 91.18 & $94.66 \pm 5$ \\
P4 Original & 100.00 & 81.53 & 69.75 & $83.76 \pm 15$ \\
P4 Corrected & 100.00 & 97.43 & 96.98 & $98.14 \pm 2$ \\
P5 Original & 100.00 & 88.56 & 81.27 & $89.94 \pm 9$ \\
P5 Corrected & 100.00 & 95.14 & 95.08 & $96.74 \pm 3$ \\
P6 Original & 100.00 & 94.26 & 88.93 & $94.40 \pm 6$ \\
P6 Corrected & 100.00 & 98.38 & 98.29 & $98.89 \pm 1$ \\
P7 Original & 100.00 & 88.69 & 84.82 & $91.17 \pm 8$ \\
P7 Corrected & 100.00 & 94.85 & 94.17 & $96.34 \pm 3$
\end{tabular}

Table 3. Myocardial Thickness Analysis on Patient Data [mm]

\begin{tabular}{l|ccc} 
Patient & \multicolumn{4}{|c}{ Target Gate Motion Corrected Not Corrected } \\
\hline P1 & 7.9 & 8.3 & 11.0 \\
P2 & 7.6 & 7.2 & 9.5 \\
P3 & 3.9 & 4.3 & 4.7 \\
P4 & 6.3 & 6.5 & 7.3 \\
P5 & 10.2 & 9.7 & 11.0 \\
P6 & 5.0 & 5.2 & 5.3 \\
P7 & 4.7 & 4.5 & 4.8 \\
\hline Avg & $6.5 \pm 2.2$ & $6.5 \pm 2.0$ & $7.7 \pm 2.8$
\end{tabular}

patient. The myocardial thickness without motion correction was between 3.9 $\mathrm{mm}$ and $11.0 \mathrm{~mm}$ which was decreased to values between $4.3 \mathrm{~mm}$ and $9.7 \mathrm{~mm}$ after motion correction.

\section{Discussion}

The PET data was corrected for motion by using respiratory gating and multiscale discontinuity-preserving optical flow. Additionally the CT based attenuation map was deformed to correspond to the different respiratory phases, see Fig. 2.

Motion of Heart. The results show that the motion correction was independent of the magnitude of motion. Thus the motion was reduced to $0.2 \mathrm{~mm}$ on patient $\mathrm{P} 1$ with $21.6 \mathrm{~mm}$ as well as on $\mathrm{P} 6$ with only $6.6 \mathrm{~mm}$ of motion. All datasets have $0.5 \mathrm{~mm}$ or less residual motion after the application of the motion 
correction scheme. Considering the voxel resolution of $3.375 \mathrm{~mm}$ on the images, we can conclude that the respiratory motion has been corrected for all practical purposes.

Correlation Coefficient. The correlation coefficients are increased to values closer to 100 as compared to before motion correction for all datasets. The improved values are almost constant for different gates e.g. the correlation was reduced by $11.79 \%$ for patient $\mathrm{P} 1$ from gate 4 to gate 8 whereas the decrease on the corrected data is only $0.35 \%$. This shows that the correction method works relatively independent of the magnitude of motion, the same result as given by the heart motion analysis. Also the standard deviation of the data becomes much smaller after motion correction as all gates approach the same respiratory phase.

Myocardial Thickness. The apparent thickness of the anterior wall due to blur caused by the respiratory motion was expected, as can been seen by comparing columns 1 and 3 of the Table3. The myocardial thickness on the motion corrected data is compareable to the values on the target gate. Thus the motion blur has been successfully reduced on the corrected image volumes. It is to be noted that in $\mathrm{P} 1$, the difference in the myocardial thickness between the corrected and the uncorrected data is around $3 \mathrm{~mm}$, or almost 1 voxel which shows that quantification based on uncorrected volumes may be significantly wrong.

\section{Conclusion}

A complete motion correction scheme comprising motion estimation on PET data, transformation of the $\mathrm{CT}$ based $\mu$-maps and reconstruction of the listmode data using these corrections was presented. The evaluation on real patient datasets with three different criteria showed that motion was successfully corrected on the PET data.

Acknowledgments. This work was partly funded by SFB 656 MoBil (projects B2, B3 and C2) of the Deutsche Forschungsgemeinschaft (DFG).

\section{References}

1. Erdi, Y., Nehmeh, S., Pan, T., Pevsner, A., Rosenzweig, K., Mageras, G., Yorke, E.D., Schoder, H., Hsiao, W., Squire, O.D., Vernon, P., Ashman, J.B., Mostafavi, H., Larson, S.M., Humm, J.L.: The CT motion quantitation of lung lesions and its impact on PET-measured SUVs. J. Nucl. Med. 45(8), 1287-1292 (2004)

2. Osman, M.M., Cohade, C., Nakamoto, Y., Marshall, L., Leal, J., Wahl, R.: Clinically significant inaccurate localization of lesions with PET/CT: frequency in 300 patients. J. Nucl. Med. 44(2), 240-243 (2003)

3. Nakamoto, Y., Chin, B., Cohade, C., Osman, M., Tatsumi, M., Wahl, R.: PET/CT: artifacts caused by bowel motion. Nucl. Med. Commun. 25(3), 221-225 (2004)

4. Dawood, M., Büther, F., Jiang, X., Schäfers, K.P.: Respiratory Motion Correction in 3D PET Data with Advanced Optical Flow Algorithms. In: IEEE Trans. Med. Imaging (in press, 2008) 
5. Dawood, M., Büther, F., Lang, N., Schober, O., Schäfers, K.P.: Respiratory gating in positron emission tomography: A quantitative comparision of different gating schemes. Medical Physics 34, 3067-3076 (2007)

6. Dawood, M., Lang, N., Jiang, X., Schäfers, K.P.: Lung motion correction on respiratory gated 3-D PET/CT images. IEEE Trans. Med. Imaging 25(4), 476-485 (2006)

7. Qiao, F., Pan, T., Clark, J.W., Mawlawi, O.R.: A motion-incorporated reconstruction method for gated PET studies. Phys. Med. Biol. 51(15), 3769-3783 (2006)

8. Mair, B.A., Gilland, D.R., Sun, J.: Estimation of images and nonrigid deformations in gated emission CT. IEEE Trans. Med. Imaging 25(9), 1130-1144 (2006)

9. Lamare, F., Carbayo, M., Cresson, T., Kontaxakis, G., Santos, A., Rest, C., Reader, A., Visvikis, D.: List-mode-based reconstruction for respiratory motion correction in PET using non-rigid body transformations. Phys. Med. Biol. 52, 5187-5204 (2007)

10. Osman, M.M., Cohade, C., Nakamoto, Y., Wahl, R.L.: Respiratory motion artifacts on pet emission images obtained using CT attenuation correction on PET-CT. Eur. J. Nucl. Med. Mol. Imaging 30(4), 603-606 (2003)

11. Vandenberghe, S., D’Asseler, Y., Van de Walle, R., Kauppinen, T., Koole, M., Bouwens, L., Van Laere, K., Lemahieu, I., Dierckx, R.A.: Iterative reconstruction algorithms in nuclear medicine. Comput. Med. Imaging Graph 25(2), 105-111 (2001)

12. Bruhn, A., Weickert, J., Schnörr, C.: Lucas/Kanade meets Horn/Schunck: Combining local and global optic flow methods. International Journal of Computer Vision 61(3), 211-231 (2005)

13. Erdi, Y.E., Nehmeh, S.A., Mulnix, T., Humm, J.L., Watson, C.C.: PET performance measurements for an LSO-based combined PET/CT scanner using the national electrical manufacturers association nu 2-2001 standard. J. Nucl. Med. 45(5), 813-821 (2004) 